\title{
MicroRNA and nephropathy: emerging concepts
}

This article was published in the following Dove Press journal: International Journal of Nephrology and Renovascular Disease 24 September 2013

Number of times this article has been viewed

\author{
Arthur CK Chung ${ }^{1,3}$ \\ Xueqing $\mathrm{Yu}^{2}$ \\ Hui Y Lan ${ }^{1,3}$ \\ 'Li Ka Shing Institute of Health \\ Sciences and Department of Medicine \\ and Therapeutics, The Chinese \\ University of Hong Kong, Hong \\ Kong, People's Republic of China; \\ ${ }^{2}$ Department of Nephrology, The \\ First Affiliated Hospital, Sun Yat-sen \\ University, Guangzhou, People's \\ Republic of China; ${ }^{3}$ CUHK Shenzhen \\ Research Institute, Shenzhen, People's \\ Republic of China
}

Correspondence: Hui Y Lan Department of Medicine and Therapeutics, Li Ka Shing Institute of Health Sciences, Rm 60I, Li Ka Shing Medical Sciences Building, The Chinese University of Hong Kong, Prince of Wales Hospital, Shatin, The New Territories, Hong Kong, People's Republic of China Tel +85237636077

Fax+852 2I45 7190

Email hylan@cuhk.edu.hk
Abstract: Micro ribonucleic acids (miRNAs) are short noncoding RNAs that inhibit gene expression through the post-transcriptional repression of their target mRNAs. Increasing evidence shows that miRNAs have emerged as key players in diverse biologic processes. Aberrant miRNA expression is also closely related to various human diseases, including kidney diseases. From clinical and experimental animal studies, emerging evidence demonstrates a critical role for miRNAs in renal pathophysiology. Renal fibrosis is the hallmark of various chronic kidney diseases and transforming growth factor beta (TGF- $\beta$ ) is recognized as a vital mediator of renal fibrosis because it can induce production of extracellular matrix proteins resulting in dysfunction of the kidneys. The relationship between TGF- $\beta$ signaling and miRNAs expression during renal diseases has been recently established. TGF- $\beta$ positively or negatively regulates expression of several miRNAs, such as miR-21, miR-192, miR-200, and miR-29. Both miR-192 and miR-2 1 are positively regulated by TGF- $\beta 1 / \mathrm{Smad} 3$ signaling and play a pathological role in kidney diseases. Conversely, members of both miR-29 and miR-200 families are negatively regulated by TGF- $\beta / \mathrm{Smad} 3$ and play a protective role in renal fibrosis by inhibiting the deposition of extracellular matrix and preventing epithelial-to-mesenchymal transition, respectively. Clinically, levels of miRNAs in circulation and urine may be potential biomarkers for detecting early stages of renal diseases and targeting miRNAs also provides promising therapeutic effects in rodent models of chronic kidney disease. However, mechanisms and roles of miRNAs under disease conditions remain to be explored. Thus, understanding the function of miRNAs in the pathogenesis of kidney diseases may offer an innovative approach for both early diagnosis and treatment of renal diseases.

Keywords: microRNAs, kidney diseases, renal fibrosis, TGF- $\beta$ signaling

\section{Introduction}

Micro ribonucleic acids (miRNAs) are small, endogenous, noncoding RNAs that bind to their respective target $m$ RNAs and recruit the RNA-induced silencing complex (RISC). The biogenesis of miRNAs includes multiple steps. Firstly, miRNAs are transcribed by RNA polymerase II or RNA polymerase III as long stem-loop primary miRNA (Pri-miR) in the nucleus. The Pri-miR is then cleaved into a double-stranded shorter miRNA precursor (Pre-miR) by RNase III enzyme Drosha and its partner DiGeorge syndrome critical region 8 (DGCR8). ${ }^{1,2}$ The Pre-miR is subsequently exported into the cytoplasm by the Ran-guanosine triphosphate (GTP) and Exportin-5. ${ }^{3}$ In the cytoplasm, Pre-miR is further cleaved into the 20-22 base pairs (bp) doublestranded RNAs (mature form) by another RNase III enzyme Dicer and this mature miRNA-miRNA duplex is unwound and the functional strand ("guide strand") is 
loaded onto the RISC. ${ }^{4}$ The mature miRNA guides the RISC complex to bind to the $3^{\prime}$ untranslated region ( $3^{\prime}$ UTR) of a target messenger RNA (mRNA), resulting in posttranscriptional gene silencing by mRNA degradation or by translation inhibition. Thus, miRNAs can inhibit gene expression via mRNA degradation, translation inhibition, or transcriptional inhibition.

Microarray assays further demonstrate that several miRNAs, including miR-192, -194, -204, -215, and -216 , are highly expressed in the kidney, compared to other organs. ${ }^{5,6}$ These studies also mark the starting point of miRNA research in kidney diseases. To date, over ten miRNAs have been reported in kidney diseases. ${ }^{7-10}$ Several comprehensive reviews about the biogenesis of miRNAs and the role of miRNAs in normal kidney have been published. ${ }^{3,4,7,10-12}$ This article highlights recent novel insights into miRNA function and the implications for miRNAs in renal disease.

\section{Role of TGF- $\beta$ I in renal pathophysiology}

Renal fibrosis and inflammation are common features of chronic kidney disease (CKD) progressing to end-stage renal failure, which is characterized by interstitial extracellular matrix (ECM), myofibroblast accumulation, and infiltration of inflammatory cells accompanied with destruction of renal tubules. ${ }^{13,14}$ It is well accepted that transforming growth factor-beta (TGF- $\beta$ ) is a master cytokine/growth factor in fibrosis and inflammation (Figure 1). ${ }^{15-20}$ TGF- $\beta$ exerts its biological effects by activating the downstream mediators Smad2 and Smad3. ${ }^{21-27}$ During fibrosis, TGF- $\beta$ is known to upregulate many fibrogenic genes, such as ECM proteins, via Smad2, Smad3, or mitogen-activated protein kinases (MAPKs). ${ }^{28-30}$ Advances in study of TGF- $\beta$ biology reveal that TGF- $\beta$ also regulates several miRNAs that are involved in renal fibrosis. TGF- $\beta 1$ is able to upregulate miR-21, miR192, miR-491-5p, miR-382, and miR-377, but downregulates the miR-29 and miR-200 families (Table 1). ${ }^{31-35}$ These TGF$\beta$-regulated miRNAs have been shown to modulate renal fibrosis (Figure 1). In addition, aberrant expression of these miRNAs is observed in mouse models of renal injury, ${ }^{31-33}$ demonstrating their critical roles in TGF- $\beta$-induced fibrosis. In this review, we focus on four groups of TGF- $\beta$-regulated miRNAs, including miR-21, miR-192, miR-200, and miR-29 as they have been shown to modulate TGF- $\beta$-induced renal fibrosis. Both miR-21 and miR-192 promote fibrosis via amplification of TGF- $\beta$ signaling while miR-29 and miR-200 families reduce fibrosis by inhibiting the ECM deposition, and preventing epithelial-to-mesenchymal transition (EMT), respectively.

\section{Role of miRNAs in TGF- $\beta$-mediated kidney diseases \\ miR-2I}

miR-21 is a prominent miRNA implicated in the genesis and progression of human cancers. ${ }^{36}$ As TGF- $\beta 1$ has been shown to upregulate miR-21 expression, ${ }^{37-39}$ the role of miR-21 in fibrotic events has been widely studied (Table 2). The functional importance of miR-21 in fibrosis was first demonstrated in heart failure. ${ }^{40}$ Its expression is upregulated in cardiac fibroblasts of failing hearts and treatment with a miR-21 antagomir in a mouse model of cardiac hypertrophy prevents interstitial fibrosis and improves cardiac function. ${ }^{40}$ Next, miR-21 was found to play a pathological role in lung fibrosis as elevation of miR-21 expression was observed in patients with idiopathic pulmonary fibrosis and in mice with bleomycin-induced lung fibrosis. ${ }^{41}$ Inhibition of miR-21 by antisense oligonucleotides reduces the severity of experimental lung fibrosis in mice. ${ }^{41}$

The pathological role of miR-21 in renal diseases has been confirmed by several models. In normal kidney tissue, expression levels of miR-21 are always low. However, its expression levels are highly elevated in both human samples of kidney diseases and animal models of CKD and acute kidney injury (AKI). ${ }^{42-47}$ In both mouse models of obstructive and diabetic nephropathy, miR-21 expression is elevated in both glomerular and tubulointerstitial area where fibrosis occurs..$^{43,45,46}$ Expression of renal miR-21 is also significantly increased after ischemia-reperfusion injury. ${ }^{44,47}$

Results of in vitro studies show that miR-21 actively participates in renal fibrosis because miR-21 positively regulates expression of ECM and $\alpha$-smooth muscle actin ( $\alpha$-SMA) $\alpha$-SMA in tubular epithelial cells (TECs) and mesangial cells (MCs) after treatment of TGF- $\beta 1$ or under diabetic conditions. ${ }^{43,46}$ In addition, overexpression of miR-21 in kidney cells promotes renal inflammation but knockdown of miR-21 reduces renal inflammation under diabetic conditions. Blocking renal miR-21 expression reduces macrophage infiltration in diseased kidneys. ${ }^{46,48}$ These results suggest that miR-21 also plays a role in promoting renal inflammation during kidney injury. ${ }^{46,48}$ The suppression of miR-21 successfully reduces fibrosis in rodent models of heart, lung, and kidney diseases. ${ }^{40,41,43}$ In a mouse model of diabetic nephropathy, inhibition of miR-21 also improves kidney function and halts the progression of renal injury, ${ }^{46}$ suggesting that targeting miR-21 should possess a therapeutic potential to ameliorate progressive kidney disease. 


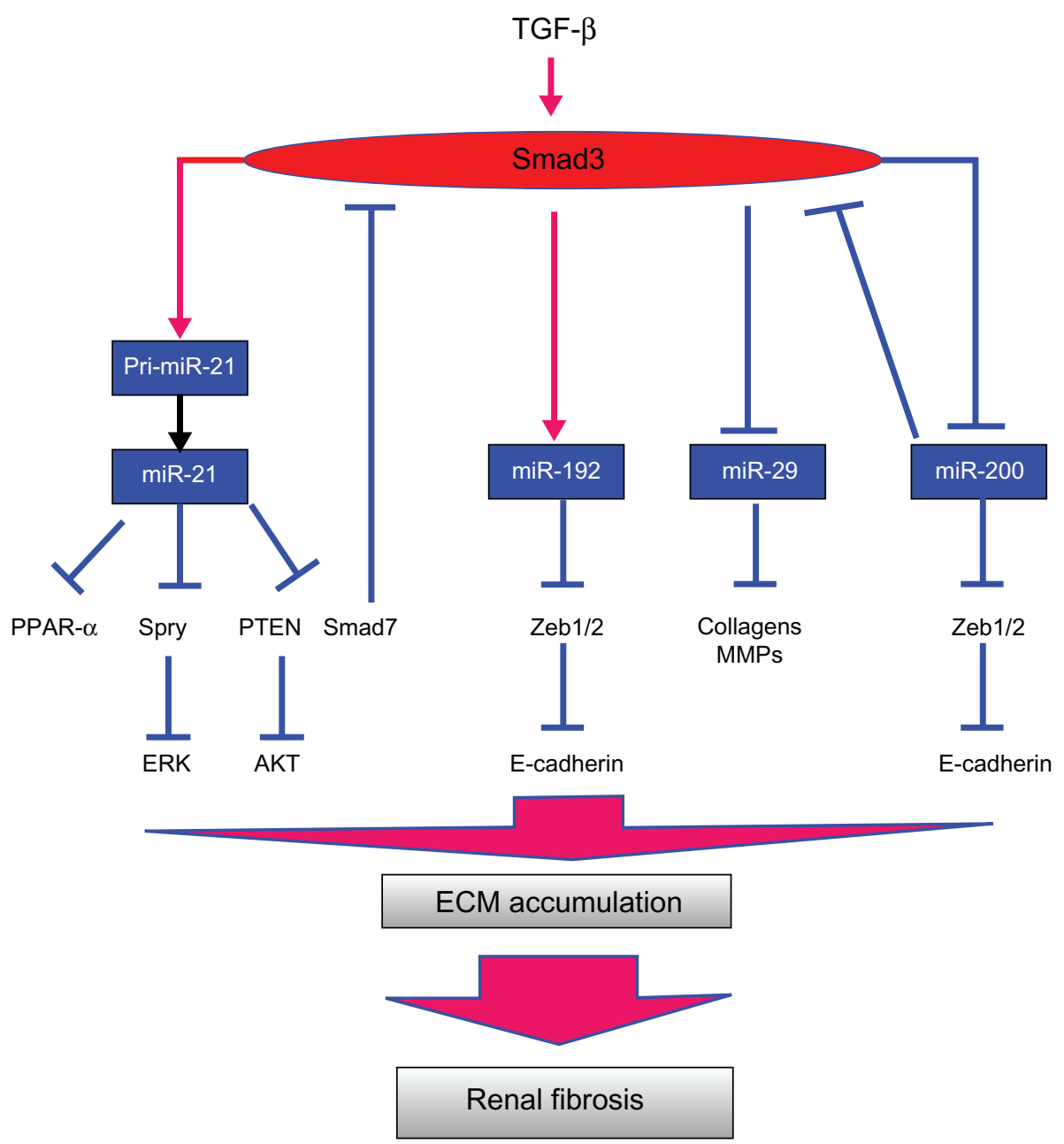

Figure I Mechanisms of TGF- $\beta$-regulated miRNAs in renal fibrosis.

Notes: TGF- $\beta$ promotes fibrosis by inducing miR-2I and miR-192 but suppressing miR-29 and miR-200 expression during renal injury. Both miR-I92 and miR-2I play a pathological role in kidney fibrosis through a feed-forward loop to amplify TGF- $\beta$ signaling and promote fibrosis. In contrast, members in miR-29 and miR-200 families play a protective role in renal fibrosis by inhibiting the deposition of extracellular matrix and preventing epithelial-to-mesenchymal transition (EMT), respectively.

Abbreviations: AKT, protein kinase B; ECM, extracellular matrix; ERK, extracellular signal-regulated kinase; miRNA, micro ribonucleic acid; MMP, matrix metalloproteinase; PPAR, peroxisome proliferator activated receptor; PTEN, phosphatase and tensin homolog;TGF- $\beta$, transforming growth factor beta; Spry, sprouty.

Table I Roles of miRNAs in animal models of kidney diseases

\begin{tabular}{|c|c|c|c|c|c|}
\hline miRNA & $\begin{array}{l}\text { Mouse model } \\
\text { of kidney diseases }\end{array}$ & $\begin{array}{l}\text { Normal } \\
\text { kidney }\end{array}$ & $\begin{array}{l}\text { Diseased } \\
\text { kidney }\end{array}$ & Results & References \\
\hline \multicolumn{6}{|c|}{ miRNAs that are induced by TGF- $\beta$} \\
\hline miR-2I & UUO, DN in $\mathrm{db} / \mathrm{db}$ mice & Low level & High level & Fibrosis and inflammation & 43,46 \\
\hline miR-192 & $\begin{array}{l}\text { UUO, STZ induced DN, } \\
\text { DN in db/db mice }\end{array}$ & Low level & High level & Fibrosis and EMT & $54-56$ \\
\hline $\operatorname{miR}-216 a$ & $\begin{array}{l}\text { STZ induced DN, DN } \\
\text { in } d b / d b \text { mice }\end{array}$ & Low level & High level & $\begin{array}{l}\text { Increases colla2 } \\
\text { expression }\end{array}$ & 93 \\
\hline miR-377 & $\begin{array}{l}\text { Spontaneous and STZ } \\
\text { induced DN }\end{array}$ & Low level & High level & Fibronectin expression & 94 \\
\hline$m i R-49 I-5 p$ & UUO (rat) & Low level & High level & Induces Par-3 degradation & 95 \\
\hline miR-382 & UUO & Low level & High level & Suppresses E-cadherin & 34 \\
\hline \multicolumn{6}{|c|}{ miRNAs that are suppressed by TGF- $\beta$} \\
\hline miR-29a,b,c & UUO & High level & Low level & Fibrosis & 63,65 \\
\hline $\begin{array}{l}\text { miR-200a,b,c } \\
\text { miR-|4I, miR-429 }\end{array}$ & $\begin{array}{l}\text { UUO STZ induced DN, } \\
\text { adenine-induced }\end{array}$ & High level & Low level & EMT & $73-75$ \\
\hline
\end{tabular}

Abbreviations: DN, diabetic nephropathy; EMT, epithelial-to-mesenchymal transition; miRNA, micro ribonucleic acid; STZ, streptozotocin; TGF- $\beta$, transforming growth factor beta; UUO, unilateral ureteral obstruction. 
Table 2 Role of miR-2I in different fibrotic diseases

\begin{tabular}{|c|c|c|c|c|}
\hline & Heart & Lung & Kidney & Liver \\
\hline Expression in normal & Low & Low & Low & NA \\
\hline $\begin{array}{l}\text { Expression } \\
\text { in diseases }\end{array}$ & High in cardiac fibroblasts & $\begin{array}{l}\text { High in pulmonary } \\
\text { fibroblasts }\end{array}$ & $\begin{array}{l}\text { High in glomeruli } \\
\text { and tubules }\end{array}$ & NA \\
\hline $\begin{array}{l}\text { Putative role } \\
\text { in fibrosis }\end{array}$ & $\begin{array}{l}\text { Induces ECM and MMP-2, } \\
\text { and TGF- } \beta \text { non-canonical } \\
\text { signaling (via Spryl to } \\
\text { de-repress ERK/MAPK) }\end{array}$ & $\begin{array}{l}\text { Induces ECM and TGF- } \beta \\
\text { canonical (via Smad7 } \\
\text { de-repression of } \\
\text { Smad2/3) signaling }\end{array}$ & $\begin{array}{l}\text { Induces ECM and MMP-2, } \\
\text { TGF- } \beta \text { canonical } \\
\text { (via Smad7 de-repression } \\
\text { of Smad } 2 / 3 \text { ) signaling }\end{array}$ & $\begin{array}{l}\text { Induces MMP-2 } \\
\text { and PTEN/Akt } \\
\text { pathway }\end{array}$ \\
\hline Signaling pathway & TGF- $\beta$ pathway & TGF- $\beta$ pathway & TGF- $\beta /$ Smad3 pathway & NA \\
\hline Pathogenic effect & $\begin{array}{l}\text { Fibrosis and } \\
\text { inflammation, } \\
\text { suppresses Spryl }\end{array}$ & $\begin{array}{l}\text { Fibrosis } \\
\text { and inflammation, } \\
\text { suppresses Smad7 }\end{array}$ & $\begin{array}{l}\text { Fibrosis and inflammation, } \\
\text { suppresses PPAR- } \alpha \text { and } \\
\text { Smad7 }\end{array}$ & $\begin{array}{l}\text { Activates hepatic } \\
\text { stellate cells via } \\
\text { PTEN/Akt signaling }\end{array}$ \\
\hline $\begin{array}{l}\text { Therapeutic effect } \\
\text { by targeting miR-2I }\end{array}$ & $\begin{array}{l}\text { Improving cardiac } \\
\text { fibrosis and function }\end{array}$ & Reducing lung fibrosis & $\begin{array}{l}\text { Suppressing renal fibrosis } \\
\text { and inflammation }\end{array}$ & NA \\
\hline Reference & 40,77 & 41 & $42-47$ & 78 \\
\hline
\end{tabular}

Abbreviations: ECM, extracellular matrix; MMP-2, matrix metalloproteinase 2; NA, not applicable; TGF- $\beta$, transforming growth factor beta; AKT, protein kinase B; ERK, extracellular signal-regulated kinase; MAPK, mitogen-activated protein kinase; PPAR, peroxisome proliferator activated receptor; PTEN, phosphatase and tensin homolog; Spry, sprouty.

miR-21 knockout (KO) mice have been successfully generated and they are phenotypically normal and exhibit normal fertility. ${ }^{42}$ As expected from the results of unilateral ureteral obstruction (UUO) and renal ischemia reperfusion injury (ISI) models, deletion of the miR-21 gene reduces tubule atrophy, fibrosis, capillary destruction, and P42/P44 MAPK pathway activation in diseased kidneys compared to wild type mice. ${ }^{42}$ Microarray analyses from miR-21 KO kidneys demonstrate the negative relationship between the presence of miR-21 and genes that are involved in lipid metabolism, fatty acid oxidation, and redox regulation. This study also shows that miR-21 promotes renal fibrosis by silencing metabolic pathways via suppressing peroxisome proliferator-activated receptor- $\alpha$ (PRAR- $\alpha$; Figure 1). ${ }^{42}$

However, the precise mechanism of how miR-21 regulates fibrosis and inflammation may be relevant for other putative target genes of miR-21. Studies in cardiac fibrosis demonstrate that Sprouty (Spry) and Phosphatase and Tensin Homolog (PTEN) are potential targets of miR-21 (Figure 1). ${ }^{40,49}$ Spry is a potent inhibitor of Ras/mitogen-activated protein kinase (MEK)/extracelluar signal-regulated kinase (ERK) and activation of ERK promotes TGF- $\beta$-dependent fibrogenic activities. ${ }^{50}$ In the heart, inhibition of miR-21 decreases ERK-MAPK activity and interstitial fibrosis. ${ }^{40}$ Suppression of PTEN by miR-21 upregulates phosphatidylinositide 3-kinases (PI3 K) and Akt activity, and subsequently induces matrix metalloproteinase (MMP)-2 expression. ${ }^{49} \mathrm{~A}$ study on diabetic kidney injury demonstrates that AKT1 substrate 1 (PRAS40), a negative regulator of Tor complex 1 (TORC1), and Smad7 are targets of miR-21. ${ }^{46,51}$ Although blocking miR-21 reduces macrophage infiltration in diseased kidneys, ${ }^{46,48}$ some studies demonstrate anti-inflammatory properties of miR-21 in macrophages by tar- geting proinflammatory programmed cell death 4 (PDCD4). ${ }^{52,53}$ Negative correlation between miR-21 and PDCD4 has been reported in TECs with induction of ischemia. ${ }^{44}$ Further studies should be done to clarify whether miR-21 regulates inflammation in a cell type-dependent fashion.

\section{miR-I 92}

miR-192 is a highly expressed miRNA in the normal kidney, compared to other organs. ${ }^{5,6}$ Several studies from rodent models of kidney diseases and cell lines demonstrate a pro-fibrotic role of miR-192 in both MCs and TECs. ${ }^{54-56}$ Elevated miR-192 expression is found in glomeruli isolated from diabetic mice. ${ }^{56}$ In cultured MCs and TECs, miR-192 expression is induced by either TGF- $\beta$ or high glucose conditions. ${ }^{55,56}$ In vitro, miR-192 also mediates TGF- $\beta$-induced collagen expression in MCs by downregulating zinc finger E-box binding homeobox $1 / 2$ (Zeb1/2) expression (Figure 1) ${ }^{56}$ Similarly, overexpression of miR-192 promotes and inhibition of miR-192 blocks TGF- $\beta 1$ induced collagen matrix expression in TECs. ${ }^{55}$ Recent studies in a mouse model of type I diabetes demonstrate that inhibition of renal miR-192 significantly induces renal expression of Zeb1/2 and suppresses expression of fibrotic markers, such as collagen, TGF- $\beta$, and fibronectin. ${ }^{54}$ More importantly, inhibition of miR-192 in vivo improves renal function by attenuating proteinuria. ${ }^{54}$ The pathological role of miR-192 in diabetic nephropathy is further confirmed by miR-192 KO mice. ${ }^{57}$ In these KO type I diabetic mice, albuminuria, proteinuria, renal fibrosis, and hypertrophy are all reduced compared to diabetic wild-type mice. ${ }^{57}$ Taken together, these studies have established a regulatory role of miR-192 in TGF- $\beta$-dependent renal pathologies observed in animal models of diabetic and obstructive nephropathy. 
As the results from animal models demonstrate a pro-fibrotic role of miR-192 in diabetic and obstructive nephropathy, ${ }^{54-56}$ the reverse is true in human nephropathy. ${ }^{58,59}$ Interestingly, miR-192 expression is reduced in human TECs after TGF- $\beta 1$ treatment or in human diseased kidneys. ${ }^{58,59}$ A recent study demonstrates that TGF- $\beta 1$ suppresses miR192 expression in human TECs and loss of miR-192 promotes fibrogenesis in diabetic nephropathy.$^{58}$ Furthermore, renal biopsy samples from diabetic patients show significantly lower miR-192. ${ }^{58}$ Reduction of miR-192 expression correlates with tubulointerstitial fibrosis and low glomerular filtration rate (GFR) in individual patients. The different findings in expression of miR-192 in human and animal models of diabetic nephropathy renders it necessary to further investigate the potential role of miR-192 and the mechanisms that regulate miR-192 expression during renal fibrosis in different species.

\section{miR-29}

The miR-29 family consists of three members that are encoded by two distinct genomic loci in both human and rodent genomes. ${ }^{60}$ As all members have the same seed binding sequence, they all bind to the same set of target genes. ${ }^{60}$ In contrast to miR-192 and miR-21, strong evidence has suggested an anti-fibrotic role for miR-29. High expression of miR-29 is found in the kidney, lung, and heart, ${ }^{60}$ and reduction of miR-29 expression is observed in animal models and human samples of fibrotic diseases in heart, lung, and kidney (Table 3). ${ }^{61-63}$

In a mouse model of obstructive nephropathy, microarrays and real-time polymerase chain reaction (PCR) assays demonstrate that all miR-29 family members (miR-29a, miR-29b, and miR-29c) are substantially reduced in fibrotic kidneys of UUO wild-type mice but significantly increased in Smad3 KO mice in which renal fibrosis was reduced. ${ }^{63}$ Reduction in expression of miR-29a and miR-29b in renal
TECs and lung and cardiac fibroblasts after treatment with TGF- $\beta 1$ confirms the negative relationship between miR29 in TGF- $\beta$ dependent fibrosis. ${ }^{61-63}$

Similar to the results in the heart in which knockdown of miR-29b relieves the suppression of fibrotic markers, ${ }^{61,64}$ overexpression of miR-29 suppresses but inhibition of miR-29 promotes expression of fibrotic markers in mouse embryonic fibroblasts and TECs under diabetic conditions, salt-induced hypertensive conditions, or after TGF- $\beta$ treatment, suggesting an anti-fibrotic effect for miR-29 (Figure 1). ${ }^{63,65,66}$ This anti-fibrotic effect of miR-29 is further confirmed by a mouse model of unilateral ureteral obstruction nephropathy. ${ }^{63}$ Gene delivery of miR-29b either before or after established obstructive nephropathy successfully blocked progressive renal fibrosis. The anti-fibrotic effects of miR-29 may be a result of its ability to inhibit TGF- $\beta$-mediated deposition of ECM. More than 20 different ECM-related genes are predicted to be miR-29 targets and some of them are induced by TGF- $\beta$ signaling. ${ }^{61}$ In conclusion, miR-29 is a downstream inhibitor of TGF- $\beta / \mathrm{Smad} 3$-mediated fibrosis and may have therapeutic potential for diseases involving fibrosis.

\section{$\operatorname{miR}-200$}

The function of miR-200 family is to maintain epithelial differentiation and this family includes miR-200a, miR-200b, miR-200c, miR-429, and miR-141. ${ }^{67}$ The miR-200 family was firstly discovered by its ability to restore an epithelial phenotype in breast cancer cell lines through the suppression of the E-cadherin transcriptional repressors ZEB1 and ZEB2 (Figure 1). ${ }^{68-71}$ Similar to the results in a mouse model of lung fibrosis, ${ }^{72}$ reduction of expression of miR-200a and miR-141 are observed in fibrotic kidneys during obstructive and diabetic nephropathy (Table 4). ${ }^{73,74}$ In vitro studies also demonstrate that members of the miR-200 family in TECs are downregulated in a Smad signaling dependent

Table 3 Role of miR-29 in different fibrotic diseases

\begin{tabular}{lllll}
\hline & Heart & Lung & Kidney & Liver \\
\hline Normal expression & High & High & High & High \\
Expression in diseases & Low in cardiac fibroblasts & Low in pulmonary fibroblasts & Low in tubules & Low \\
Putative role in fibrosis & Reducing ECM and MMP-2 & Reducing ECM expression & Reducing ECM and MMP-2 & Reducing ECM expression \\
Signaling pathway & Suppressed by TGF- $\beta$ & Suppressed by TGF- $\beta$ & Suppressed by & Suppressed by TGF- $\beta$ \\
& pathway & pathway & TGF- $\beta /$ Smad3 pathway & and NF-KB pathways \\
Pathogenic effect & Induces fibrosis & Induces fibrosis & Induces fibrosis & Induces fibrosis \\
Therapeutic effect & & Overexpression of miR-29 & Overexpression & Overexpression of \\
& & reduces the severity & of miR-29 suppresses & miR-29 suppresses \\
& & of lung fibrosis & renal fibrosis & hepatic fibrosis \\
Reference & 61 & 62,79 & 63,66 & $80-82$ \\
\hline
\end{tabular}

Abbreviations: ECM, extracellular matrix; MMP-2, matrix metalloproteinase 2; NF-אB, nuclear factor kappa-light-chain-enhancer of activated B cells; TGF- $\beta$, transforming growth factor beta. 
manner after TGF- $\beta$ treatment. ${ }^{73,74}$ However, another study shows opposite results; that renal expression of miR-200s is increased in mice subjected to UUO. ${ }^{75}$ Regardless of the discrepancy in miR-200 expression in fibrotic kidneys, the anti-fibrotic role of miR-200 family is confirmed by gene delivery of miR-200b in fibrotic kidneys. The results show that the elevation of collagens and fibronectin in obstructed kidneys can be suppressed by a single injection of a miR200 b precursor. $^{75}$

The maintenance of epithelial differentiation and prevention of EMT may be the mechanism in which miR200 suppresses fibrosis. ${ }^{71}$ In epithelial cells, members of the miRNA-200 family inhibit the E-cadherin transcriptional repressors ZEB1 and ZEB2, which were previously implicated in EMT and tumor metastasis. ${ }^{68-71}$ These miRNAs are also markedly downregulated in cells that have undergone EMT in response to TGF- $\beta,{ }^{68-71}$ suggesting that TGF- $\beta$ regulates the expression of these miRNAs to promote EMT in tumor cells. In response to renal injury, it is generally believed that proximal TECs may undergo EMT and contribute to renal fibrosis. ${ }^{14}$ However, the notion that EMT contributes to renal fibrosis has recently been challenged. More research is needed to clarify the role of miR-200 in kidney diseases.

\section{Regulatory mechanisms of TGF- $\beta I$ in expression of fibrosis-related miRNAs}

The exact mechanism of how TGF- $\beta$ signaling regulates miRNA expression during kidney diseases is still ongoing. TGF- $\beta$ signaling promotes synthesis of fibrosis-related miRNAs either by enhancing posttranscriptional processing of the primary miRNA transcript, or by increasing transcription. It has been demonstrated that TGF- $\beta$ signaling is able to promote the processing of primary transcripts of some miRNAs into their active form by the Drosha complex. ${ }^{37}$
Receptor-Smads, such as Smad3, physically interact with subunits of the Drosha complex to promote the processing of pri-miR-21 into mature miR-21 (Figure 1). Davis et al also demonstrate that a consensus sequence (R-SBE) exists within the stem region of the primary transcripts of TGF- $\beta$ regulated-miRs (pri-T-miRs). ${ }^{39}$ Direct binding of Smads to the R-SBE triggers the TGF- $\beta$-induced recruitment of Drosha and DGCR8 to pri-T-miRs and promotes the processing of pri-T-miRs. $^{39}$

Our laboratory also demonstrated that TGF- $\beta / \mathrm{Smad} 3$ signaling regulates the transcription of miR-21, miR-192, and the miR-29 family during renal diseases (Figure 1). ${ }^{43,55,63}$ This notion is firstly supported by the results from rodent models of obstructive and remnant kidney diseases induced in mice lacking Smad3, Smad7, or having conditional $\mathrm{KO}$ of Smad2 or overexpressing renal Smad7..$^{33,43,55,63}$ Results from in vitro studies also confirm that TGF- $\beta$ suppresses miR-29 expression but induces miR-21 and miR-192 expression via the Smad3-dependent mechanism as determined in MCs and TECs overexpressing Smad7, or knocking down Smad2 or Smad3 and in Smad2 or Smad3 KO mouse embryonic fibroblasts. ${ }^{33,43,55,63}$ In addition, Smad3 regulates the expression of these miRNAs by physically interacting with Smad binding site (SBE) located in their promoters. ${ }^{43,55,63}$ Binding of Smad3 on SBE in the promoters may either increase transcription and post-transcriptional processing of miRNAs, such as miR-21 and miR-192, or inhibit their transcription, such as for miR-29b. ${ }^{43,55,63}$ In addition, Smad7, an inhibitory Smad, protects kidneys from fibrosis based on its ability to regulate $\mathrm{TGF}-\beta / \mathrm{Smad} 3$-mediated miRNAs via maintaining renal miR-29b but suppressing miR-192 and miR-21.22,33,55

More importantly, miRNAs can also regulate TGF- $\beta$ / Smad3 signaling (Figure 1). As TGF- $\beta$ induces miR-21 expression during renal injury, the elevation of miR-21 suppresses Smad7 expression and, in turn, promotes the TGF- $\beta$ signaling. ${ }^{41,46}$ Thus, miR-21 may act as a feed-forward loop

Table 4 Role of miR-200 family members in different fibrotic diseases

\begin{tabular}{lll}
\hline & Lung & Kidney \\
\hline Normal expression & High & High \\
Expression in diseases & Low & Low \\
$\begin{array}{l}\text { Putative role } \\
\text { in fibrosis }\end{array}$ & Suppress & Suppress TGF- $\beta$ pathway \\
Signaling pathway & TGF- $\beta$-dependent EMT & and TGF- $\beta$-dependent EMT \\
Pathogenic effect & Suppressed by TGF- $\beta$ pathway & Suppressed by TGF- $\beta$ pathway \\
Therapeutic effect & EMT and fibrosis & EMT and fibrosis \\
& Overexpression of miR-200 & Overexpression of miR-200 \\
Reference & suppresses pulmonary fibrosis & suppresses renal fibrosis \\
\hline
\end{tabular}

Abbreviations: EMT, epithelial-to-mesenchymal transition; TGF- $\beta$, transforming growth factor beta. 
to amplify TGF- $\beta$ signal during renal injury. Activation of ERK/MAP kinase signaling in fibroblasts may be another mechanism by which miR-21 induces renal fibrosis. ${ }^{40}$ Furthermore, both TGF- $\beta 1$ and TGF- $\beta 2$ suppress miR-200a expression in renal cells. ${ }^{74}$ Since TGF- $\beta 2$ is one of the target genes for miR-200a, the reduction of miR-200 expression after treatment of TGF- $\beta$ is associated with an elevation in TGF- $\beta 2$ expression. On the other hand, overexpression of miR-200a also inhibits both Smad3 activity and TGF- $\beta 1$ induced fibrosis. ${ }^{74}$ These results reveal a possible feedback between TGF- $\beta 2$ and miR-200a. Similar to miR-200, miR-29 is predicted to inhibit TGF- $\beta 2 .{ }^{61}$ Thus, miR-29 may exert its anti-fibrotic effects through inhibition of TGF- $\beta$ signaling.

In addition, a recent study demonstrates that TGF- $\beta$ induces a crosstalk circuit between p53 and miR-192 related to the pathogenesis of diabetic nephropathy. ${ }^{57}$ Expression levels of TGF- $\beta 1$, p53, and miR-192 are known to be elevated in expanded glomeruli of diabetic mice..$^{54,56,57}$ Interestingly, inhibition of miR-192 function in vivo is able to suppress p53 expression in the renal cortex of control and streptozotocin injected diabetic mice. ${ }^{57}$ This positive relationship between miR-192 and renal expression of TGF- $\beta$ and p53 is confirmed by the miR-192 KO type I diabetic mice. ${ }^{57}$ Using promoter assay studies in vitro, TGF- $\beta$ is able to induce activation of miR-192 and p53. ${ }^{57}$

All the above results suggest the essential roles of miRNAs in TGF- $\beta$-induced renal fibrosis and the complexity between TGF- $\beta /$ Smads signaling and miRNAs under pathophysiological conditions.

\section{Comparison of the role of miRNAs in other fibrotic diseases}

In addition to renal fibrosis, miRNAs also participate in fibrotic diseases in other organs. To date, about 40 miRNAs have been reported to be related to fibrosis in various organs, the details of which have been discussed elsewhere. ${ }^{76}$ In this review, we compare the four groups of miRNAs in fibrotic diseases of heart, lung, kidney, and liver (Tables 2-4). Pro-fibrotic miRNAs, such as miR-21, and anti-fibrotic miRNAs, such as miR-29 and miR-200 families, are dysregulated in these organs as fibrosis occurs. ${ }^{40-47,61-63,66,77-82}$

These miRNAs directly induce or protect against fibrosis via four different mechanisms. First, regulating the TGF- $\beta$ pathway is a key mechanism for the fibrotic response of miRNAs during fibrosis (Table 2). ${ }^{40-47}$ In the lung and kidney, miR-21 upregulates TGF- $\beta$ canonical signaling by suppressing Smad7. However, miR-21 in the heart induces TGF- $\beta$ non-canonical signaling by targeting Spry1 for derepression of ERK/MAPK. In addition, miR-200 is capable of suppressing expression of TGF- $\beta 2$ to exert its anti-fibrotic effect in kidney disease (Table 4). ${ }^{74}$

Regulating ECM proteins or enzymes that are involved in ECM remodeling is another vital mechanism for miRNAs during fibrosis. miR-29 is the best characterized direct regulator of ECM synthesis and has been reported in many major fibrotic diseases in the heart, lung, liver, and kidney (Table 3). ${ }^{61-63,66,79-82}$ Indeed, a large number of ECM and ECM-modulating genes, such as COL1A1, COL3A1, COL4A1, COL5A1, COL5A2, COL5A3, COL7A1, COL8A1, and $M M P 2$, have been validated as direct targets of miR-29 by reporter gene assays. ${ }^{61,66,79}$ Expression of miR-29 has been reported to be regulated by TGF- $\beta$ signaling in the heart, lung, liver, and kidney. ${ }^{61-63,66,79-82}$ In addition, the nuclear factor kappa-light-chain-enhancer of activated B cells (NF- $\mathrm{B}$ ) pathway is also involved in suppression of hepatic miR-29 expression. ${ }^{80-82}$ miR-21 is another fibrotic miRNA found in the heart, lung, and kidney although its upregulatory effects may be indirect. ${ }^{40-47,77,78}$ Thirdly, regulating EMT may be also a mechanism by which miRNAs are involved in the fibrotic process. miR-200 members and miR-192 are closely related to EMT because they negatively regulate the E-cadherin transcriptional repressors ZEB1 and ZEB2 (Table 4). ${ }^{56,68-71}$ Overexpression of miR-200 members or inhibition of miR192 prevents TGF- $\beta$-dependent EMT and reduces expression of ECM proteins. ${ }^{56,68-71}$ Finally, some miRNAs may exert their regulatory roles in inducing proliferation and resistance to apoptosis during the fibrotic process, ${ }^{76}$ although no direct evidence was found during the process of fibrosis.

\section{Clinical implications of miRNAs in kidney diseases \\ Therapeutic potential}

As we discussed previously, miRNAs play essential roles in renal injury and miRNAs are always located in the cytoplasm of cells. These two factors support the therapeutic potential of miRNAs in kidney diseases. Furthermore, sequence complementarity provides a possible and specific approach to design a drug that precisely targets a single miRNA. Recent advances in chemical engineering allow the development of chemically modified oligonucleotides that are stable in the circulation and can freely enter cells to bind to specific miRNA and silence it. ${ }^{10}$ Conventional construction of overexpression or short hairpin (sh)RNA plasmids allow an alternative to restore or suppress miRNA transcription. ${ }^{43,63}$ In experimental kidney disease models, restoring miR-29 and miR-200 families, or inhibition of 
miR-21 and miR-192, are able to prevent or inhibit renal fibrosis (Table 5). ${ }^{33,43,46,54,63,75}$ Inhibition of miRNAs by chemical-engineered oligonucleotides has also been applied to other miRNAs. ${ }^{10}$ Thus, application of miRNAs or its inhibitor in the treatment of kidney disease offers a novel and effective therapeutic approach to combat CKD.

Major obstacles to the therapeutic use of miRNAs are the delivery method and safety concerns. To date, systemic delivery of chemical-engineered oligonucleotides has been widely used for inhibiting miRNA function. ${ }^{10}$ However, this method may also suppress the function of miRNAs in organs other than the diseased one. Gene delivery systems to regulate miRNA expression in specific organs have also been developed. ${ }^{62,83}$ For instance, ultrasound-microbubblemediated gene transfer developed in our laboratory is capable of delivering miRNA overexpression or knockdown plasmids specifically into living kidneys. ${ }^{33,43,46,63,83}$ Controlling transgene expression at the desired therapeutic levels to minimize side-effects is essential to the success of gene therapy. To avoid any undesirable side-effects caused by overdoses of overexpression or inhibition of miRNA expression, optimal dosages of miRNAs need to clearly be determined and controlled.

The risks of off-target effects and nonspecific immune responses also need to be considered and investigated. For example, the major obstacle to using miR-21 as a therapeutic agent for fibrotic diseases is that downregulation of miR-21 expression induces cell death. ${ }^{43,44,84}$ Strong pro-apoptotic effects of miR-29b may also hinder the development of miR$29 \mathrm{~b}$ gene therapy as overexpression of miRNA-29b induces apoptosis in multiple myeloma cells. ${ }^{85}$ Thus, miRNA therapeutics still require the development of specific and consistent delivery systems that target precise cells and organs.

\section{Biomarkers}

Another potential application of miRNAs is as excellent biomarkers of kidney diseases. Recently, circulating nucleic acids have emerged as new biomarkers and many studies are implying that circulating miRNAs are potential biomarkers for cancer growth and organ injury because miRNAs are stable and tissue specific as well as the fact that they can be identified and quantified. ${ }^{86}$ Several studies confirm the fact that miRNAs are released into urine or blood during kidney disease (Table 5). For instance, circulating miR-21 levels have been demonstrated to be significantly higher in patients with severe interstitial fibrosis and tubular atrophy. ${ }^{87} \mathrm{~A}$ recent study shows that a total of 27 miRNAs are present at significantly different levels in urine from patients at different stages of diabetic nephropathy. ${ }^{88}$ More importantly, these miRNAs have previously been shown to play essential roles in signaling pathways of renal fibrosis during diabetic kidney disease. Another study demonstrates that urinary miR-93 levels are related to glomerular scarring while urinary levels of miR-29b and $\mathrm{miR}-29 \mathrm{c}$ are correlated with proteinuria and renal function in immunoglobulin A ( $\operatorname{Ig} \mathrm{A}$ ) nephropathy. ${ }^{89}$ These studies demonstrate that miRNAs are not restricted to the kidney only during disease or development. Future studies should focus on the identification of patterns of miRNAs that are released into the urine or blood by damaged kidneys.

\section{Summary and perspectives}

Over the past two decades, the progress in discovering miRNAs and characterizing their functions in kidney diseases has been rapid. However, understanding the specific role of miRNAs in renal pathophysiology is still limited. One of the critical issues that hinders the progress of miRNAs in renal research is how to accurately identify miRNA targets because

Table 5 Summary of clinical implications of miRNAs

\begin{tabular}{|c|c|c|c|c|c|}
\hline \multirow[t]{2}{*}{ miRNA } & \multicolumn{2}{|c|}{$\begin{array}{l}\text { Therapeutic applications } \\
\text { (animal models) } \\
\end{array}$} & \multicolumn{2}{|c|}{ Biomarkers (patients) } & \multirow[t]{2}{*}{ References } \\
\hline & Model & Side effect & Type & Correlation & \\
\hline \multicolumn{6}{|l|}{ miR-29 } \\
\hline Overexpression & UUO & Pro-apoptotic & Urinary & $\begin{array}{l}\text { Proteinuria and renal } \\
\text { function }\end{array}$ & $33,43,46,63,85,89$ \\
\hline \multicolumn{6}{|l|}{ miR-200 } \\
\hline Overexpression & UUO & & & & 75 \\
\hline \multicolumn{6}{|l|}{ miR-2I } \\
\hline Knockdown & UUO, DN & Pro-apoptotic & Plasma & $\begin{array}{l}\text { Interstitial fibrosis and } \\
\text { tubular atrophy }\end{array}$ & $43,44,46,84$ \\
\hline \multicolumn{6}{|l|}{ miR-192 } \\
\hline Knockdown & UUO, DN & & & & 54 \\
\hline miR-93 & & & Urinary & Glomerular scarring & 89 \\
\hline
\end{tabular}

Abbreviations: DN, diabetic nephropathy; miRNA, micro ribonucleic acid; UUO, unilateral ureteral obstruction. 
one miRNA is capable of regulating multiple target genes. To date, target prediction programs provide us with a large number of potential miRNA targets. However, the overlap between algorithms is so minimal that only a small portion of these targets can be validated experimentally. Although conservation of the $3^{\prime}$ UTR among species is included, the number of targets predicted is still high for validation. In addition, the power of miRNAs also depends on their ability to target multiple genes that contribute to a pathway or phenotype. This also increases the difficulty in searching for real targets of miRNAs. However, high-throughput validation and proteomic analysis are possible approaches to enable us to identify miRNA targets.

Understanding the regulation of miRNA expression will be another challenge for miRNA research because one miRNA expression can be regulated by many different mediators or pathways. Firstly, miRNAs in a given miRNA cluster may show the same expression pattern. However, some cluster members do not follow the same pattern and exhibit different expression patterns. ${ }^{90}$ Furthermore, some miRNAs may be encoded from two or more distinct genomic loci, such as miR-29b; ${ }^{60}$ it is still not known how to control miR-29b expression from two genomic loci. Intronic miRNAs do not always have the same expression pattern as their host gene. ${ }^{91}$ Furthermore, sometimes both strands of the miRNA are coexpressed and they usually target different genes. ${ }^{92}$ Further studies are required to understand the mechanism regarding how to regulate miRNA expression. Recent technology advances in deep sequencing may provide us a comprehensive view of gene expression patterns and quantify transcript levels. This information may allow us to correlate expression of miRNAs and target transcripts.

Understanding the pathophysiological role of a specific miRNA in the kidney is not as easy as previous thought because the kidney is composed of several types of cells and these cell types may respond differentially to miRNAs in various renal diseases. Identifying the cell type(s) that expresses the miRNA in a particular disease condition may enable us to understand the role of miRNAs during disease progression. To overexpress or inhibit the function of a specific miRNA in a particular cell type will be beneficial to our understanding of disease mechanisms. Thus, transgenic animal models with conditional overexpression or knockdown of a particular miRNA may provide the best model to study the function and regulation of miRNAs in renal diseases. This approach will improve our knowledge of the role of miRNAs in renal pathology and enable us to find new treatments for halting renal fibrosis and dysfunction. However, identifying the functional significance of a change in miRNA regulation and its target gene(s) may be difficult because a single miRNA can regulate several target proteins and a single protein can be regulated by several miRNAs.

Finally, miRNAs act as important downstream effectors of TGF- $\beta /$ Smad signaling during renal fibrosis. Advances in our understanding of the specific role of miRNAs involved in TGF- $\beta$ /Smad signaling during renal fibrosis should provide us an effective, alternative strategy to ameliorate disease progression.

\section{Acknowledgments}

This work was supported by grants from Major State Basic Research Development Program of China (973 program, No 2012CB517700 to HYL) and National Natural Science Foundation of China (General Program 81170681 to ACC and Major Program 81130012 to XY); the Research Grant Council of Hong Kong (RGC GRF 468711, 469110, CUHK5/CRF/09, and CUHK3/CRF/12R to HYL; GRF 463612, 464010, 763908, and 764109 to ACC); the Focused Investment Scheme A and B and direct grants from the Chinese University of Hong Kong (2011.1.076, 2012.1.021 to ACC); 2011 Research Grant from Hong Kong Society of Nephrology (6903213 to ACC); Municipal Science and Technology R\&D funding of basic research, Shenzhen (Major Program JC201104220290 A to HYL and General Program C.02.12.00501 to ACC).

\section{Disclosure}

The authors report no conflicts of interest in this work.

\section{References}

1. Gregory RI, Yan KP, Amuthan G, et al. The Microprocessor complex mediates the genesis of microRNAs. Nature. 2004;432(7014):235-240.

2. Lee $Y$, Ahn C, Han J, et al. The nuclear RNase III Drosha initiates microRNA processing. Nature. 2003;425(6956):415-419.

3. Du T, Zamore PD. microPrimer: the biogenesis and function of microRNA. Development. 2005;132(21):4645-4652.

4. Filipowicz W. RNAi: the nuts and bolts of the RISC machine. Cell. 2005;122(1):17-20.

5. Tian Z, Greene AS, Pietrusz JL, Matus IR, Liang M. MicroRNA-target pairs in the rat kidney identified by microRNA microarray, proteomic, and bioinformatic analysis. Genome Res. 2008;18(3):404-411.

6. Sun Y, Koo S, White N, et al. Development of a micro-array to detect human and mouse microRNAs and characterization of expression in human organs. Nucleic Acids Res. 2004;32(22):e188.

7. Bhatt K, Mi QS, Dong Z. microRNAs in kidneys: biogenesis, regulation, and pathophysiological roles. Am J Physiol Renal Physiol. 2011;300(3): F602-F610.

8. Li JY, Yong TY, Michael MZ, Gleadle JM. Review: the role of microRNAs in kidney disease. Nephrology (Carlton). 2010;15(6):599-608.

9. Kato M, Arce L, Natarajan R. MicroRNAs and their role in progressive kidney diseases. Clin J Am Soc Nephrol. 2009;4(7):1255-1266. 
10. Lorenzen JM, Haller H, Thum T. MicroRNAs as mediators and therapeutic targets in chronic kidney disease. Nat Rev Nephrol. 2011;7(5):286-294.

11. Wessely O, Agrawal R, Tran U. MicroRNAs in kidney development: lessons from the frog. RNA Biol. 2010;7(3):296-299.

12. Saal S, Harvey SJ. MicroRNAs and the kidney: coming of age. Curr Opin Nephrol Hypertens. 2009;18(4):317-323.

13. Böttinger EP. TGF-beta in renal injury and disease. Semin Nephrol. 2007;27(3):309-320.

14. Liu Y. Cellular and molecular mechanisms of renal fibrosis. Nat Rev Nephrol. 2011;7(12):684-696.

15. Roberts AB. Molecular and cell biology of TGF-beta. Miner Electrolyte Metab. 1998;24(2-3):111-119.

16. Wang W, Huang XR, Li AG, et al. Signaling mechanism of TGF-beta1 in prevention of renal inflammation: role of Smad7. J Am Soc Nephrol. 2005;16(5):1371-1383

17. Lan HY, Chung AC. Transforming growth factor- $\beta$ and Smads. Contrib Nephrol. 2011;170:75-82.

18. Meng XM, Huang XR, Xiao J, et al. Disruption of Smad4 impairs TGF- $\beta /$ Smad 3 and Smad 7 transcriptional regulation during renal inflammation and fibrosis in vivo and in vitro. Kidney Int. 2012;81(3):266-279.

19. Meng XM, Huang XR, Xiao J, et al. Diverse roles of TGF- $\beta$ receptor II in renal fibrosis and inflammation in vivo and in vitro. $J$ Pathol. 2012;227(2):175-188.

20. Meng XM, Huang XR, Chung AC, et al. Smad2 protects against TGFbeta/Smad3-mediated renal fibrosis. J Am Soc Nephrol. 2010;21(9): 1477-1487.

21. Massagué J, Chen YG. Controlling TGF-beta signaling. Genes Dev. 2000;14(6):627-644.

22. Miyazono K. Positive and negative regulation of TGF-beta signaling. J Cell Sci. 2000;113(Pt 7):1101-1109.

23. Meng XM, Chung AC, Lan HY. Role of the TGF- $\beta /$ BMP-7/Smad pathways in renal diseases. Clin Sci. 2013;124(4):243-254.

24. Chung AC, Zhang H, Kong YZ, et al. Advanced glycation end-products induce tubular CTGF via TGF-beta-independent Smad3 signaling. J Am Soc Nephrol. 2010;21(2):249-260.

25. Chen HY, Huang XR, Wang W, et al. The protective role of Smad7 in diabetic kidney disease: mechanism and therapeutic potential. Diabetes. 2011;60(2):590-601.

26. Zhou L, Fu P, Huang XR, et al. Mechanism of chronic aristolochic acid nephropathy: role of Smad3. Am J Physiol Renal Physiol. 2010;298(4): F1006-F1017.

27. Li R, Lan HY, Chung AC. Distinct roles of Smads and microRNAs in TGF- $\beta$ signaling during kidney diseases. Hong Kong Journal of Nephrology. 2013;15(1):14-21.

28. Schnaper HW, Hayashida T, Hubchak SC, Poncelet AC. TGF-beta signal transduction and mesangial cell fibrogenesis. Am J Physiol Renal Physiol. 2003;284(2):F243-F252.

29. Hoffman BB, Sharma K, Ziyadeh FN. Potential role of TGF-beta in diabetic nephropathy. Miner Electrolyte Metab. 1998;24(2-3):190-196.

30. Chung AC, Huang XR, Zhou L, Heuchel R, Lai KN, Lan HY. Disruption of the Smad7 gene promotes renal fibrosis and inflammation in unilateral ureteral obstruction (UUO) in mice. Nephrol Dial Transplant. 2009;24(5):1443-1454.

31. Kantharidis P, Wang B, Carew RM, Lan HY. Diabetes complications: the microRNA perspective. Diabetes. 2011;60(7):1832-1837.

32. Lan HY, Chung AC. TGF- $\beta /$ Smad signaling in kidney disease. Semin Nephrol. 2012;32(3):236-243.

33. Chung AC, Dong Y, Yang W, Zhong X, Li R, Lan HY. Smad7 suppresses renal fibrosis via altering expression of TGF- $\beta / \mathrm{Smad} 3$-regulated microRNAs. Mol Ther. 2013;21(2):388-398.

34. Kriegel AJ, Liu Y, Cohen B, Usa K, Liu Y, Liang M. MiR-382 targeting of kallikrein 5 contributes to renal inner medullary interstitial fibrosis. Physiol Genomics. 2012;44(4):259-267.

35. Kriegel AJ, Fang Y, Liu Y, et al. MicroRNA-target pairs in human renal epithelial cells treated with transforming growth factor beta 1: a novel role of miR-382. Nucleic Acids Res. 2010;38(22):8338-8347.
36. Jazbutyte V, Thum T. MicroRNA-21: from cancer to cardiovascular disease. Curr Drug Targets. 2010;11(8):926-935.

37. Davis BN, Hilyard AC, Lagna G, Hata A. SMAD proteins control DROSHA-mediated microRNA maturation. Nature. 2008;454(7200): $56-61$.

38. Zavadil J, Narasimhan M, Blumenberg M, Schneider RJ. Transforming growth factor-beta and microRNA: mRNA regulatory networks in epithelial plasticity. Cells Tissues Organs (Print). 2007;185(1-3): $157-161$

39. Davis BN, Hilyard AC, Nguyen PH, Lagna G, Hata A. Smad proteins bind a conserved RNA sequence to promote microRNA maturation by Drosha. Mol Cell. 2010;39(3):373-384.

40. Thum T, Gross C, Fiedler J, et al. MicroRNA-21 contributes to myocardial disease by stimulating MAP kinase signalling in fibroblasts. Nature. 2008;456(7224):980-984.

41. Liu G, Friggeri A, Yang Y, et al. miR-21 mediates fibrogenic activation of pulmonary fibroblasts and lung fibrosis. J Exp Med. 2010;207(8): 1589-1597.

42. Chau BN, Xin C, Hartner J, et al. MicroRNA-21 promotes fibrosis of the kidney by silencing metabolic pathways. Sci Transl Med. 2012;4(121): $121 \mathrm{ra} 18$.

43. Zhong X, Chung AC, Chen HY, Meng XM, Lan HY. Smad3-mediated upregulation of miR-21 promotes renal fibrosis. J Am Soc Nephrol. 2011;22(9):1668-1681.

44. Godwin JG, Ge X, Stephan K, Jurisch A, Tullius SG, Iacomini J. Identification of a microRNA signature of renal ischemia reperfusion injury. Proc Natl Acad Sci U S A. 2010;107(32):14339-14344.

45. Wang J, Gao Y, Ma M, et al. Effect of miR-21 on renal fibrosis by regulating MMP-9 and TIMP1 in kk-ay diabetic nephropathy mice. Cell Biochem Biophys. 2013.

46. Zhong X, Chung AC, Chen HY, et al. miR-21 is a key therapeutic target for renal injury in a mouse model of type 2 diabetes. Diabetologia. 2013;56(3):663-674.

47. $\mathrm{Xu} \mathrm{X}$, Kriegel AJ, Liu Y, et al. Delayed ischemic preconditioning contributes to renal protection by upregulation of miR-21. Kidney Int. 2012;82(11):1167-1175.

48. Zarjou A, Yang S, Abraham E, Agarwal A, Liu G. Identification of a microRNA signature in renal fibrosis: role of miR-21. Am J Physiol Renal Physiol. 2011;301(4):F793-F801.

49. Roy S, Khanna S, Hussain SR, et al. MicroRNA expression in response to murine myocardial infarction: miR-21 regulates fibroblast metalloprotease-2 via phosphatase and tensin homologue. Cardiovasc Res. 2009;82(1):21-29.

50. Ding Q, Gladson CL, Wu H, Hayasaka H, Olman MA. Focal adhesion kinase (FAK)-related non-kinase inhibits myofibroblast differentiation through differential MAPK activation in a FAK-dependent manner. J Biol Chem. 2008;283(40):26839-26849.

51. Dey N, Das F, Mariappan MM, et al. MicroRNA-21 orchestrates high glucose-induced signals to TOR complex 1 , resulting in renal cell pathology in diabetes. J Biol Chem. 2011;286(29):25586-25603.

52. Sheedy FJ, Palsson-McDermott E, Hennessy EJ, et al. Negative regulation of TLR4 via targeting of the proinflammatory tumor suppressor PDCD4 by the microRNA miR-21. Nat Immunol. 2010;11(2): 141-147.

53. Merline R, Moreth K, Beckmann J, et al. Signaling by the matrix proteoglycan decorin controls inflammation and cancer through PDCD4 and MicroRNA-21. Sci Signal. 2011;4(199):ra75.

54. Putta S, Lanting L, Sun G, Lawson G, Kato M, Natarajan R. Inhibiting microRNA-192 ameliorates renal fibrosis in diabetic nephropathy. J Am Soc Nephrol. 2012;23(3):458-469.

55. Chung AC, Huang XR, Meng X, Lan HY. miR-192 mediates TGFbeta/Smad3-driven renal fibrosis. J Am Soc Nephrol. 2010;21(8): 1317-1325.

56. Kato M, Zhang J, Wang M, et al. MicroRNA-192 in diabetic kidney glomeruli and its function in TGF-beta-induced collagen expression via inhibition of E-box repressors. Proc Natl Acad Sci U S A. 2007;104(9): $3432-3437$ 
57. Deshpande SD, Putta S, Wang $M$, et al. Transforming growth factor- $\beta$ induced cross talk between p53 and a microRNA in the pathogenesis of diabetic nephropathy. Diabetes. 2013.

58. Krupa A, Jenkins R, Luo DD, Lewis A, Phillips A, Fraser D. Loss of MicroRNA-192 promotes fibrogenesis in diabetic nephropathy. $\mathrm{J} \mathrm{Am}$ Soc Nephrol. 2010;21(3):438-447.

59. Wang B, Herman-Edelstein M, Koh P, et al. E-cadherin expression is regulated by miR-192/215 by a mechanism that is independent of the profibrotic effects of transforming growth factor-beta. Diabetes 2010;59(7):1794-1802.

60. Kriegel AJ, Liu Y, Fang Y, Ding X, Liang M. The miR-29 family: genomics, cell biology, and relevance to renal and cardiovascular injury. Physiol Genomics. 2012;44(4):237-244.

61. van Rooij E, Sutherland LB, Thatcher JE, et al. Dysregulation of microRNAs after myocardial infarction reveals a role of miR-29 in cardiac fibrosis. Proc Natl Acad Sci U S A. 2008;105(35):13027-13032.

62. Xiao J, Meng XM, Huang XR, et al. miR-29 inhibits bleomycin-induced pulmonary fibrosis in mice. Mol Ther. 2012;20(6):1251-1260.

63. Qin W, Chung AC, Huang XR, et al. TGF- $\beta /$ Smad 3 signaling promotes renal fibrosis by inhibiting miR-29. J Am Soc Nephrol. 2011;22(8): $1462-1474$.

64. Ye Y, Hu Z, Lin Y, Zhang C, Perez-Polo JR. Downregulation of microRNA-29 by antisense inhibitors and a PPAR-gamma agonist protects against myocardial ischaemia-reperfusion injury. Cardiovasc Res. 2010;87(3):535-544.

65. Du B, Ma LM, Huang MB, et al. High glucose down-regulates miR-29a to increase collagen IV production in HK-2 cells. FEBS Lett. 2010;584(4):811-816.

66. Liu Y, Taylor NE, Lu L, et al. Renal medullary microRNAs in Dahl salt-sensitive rats: miR-29b regulates several collagens and related genes. Hypertension. 2010;55(4):974-982.

67. Howe EN, Cochrane DR, Richer JK. The miR-200 and miR-221/222 microRNA families: opposing effects on epithelial identity. J Mammary Gland Biol Neoplasia. 2012;17(1):65-77.

68. Gregory PA, Bert AG, Paterson EL, et al. The miR-200 family and miR-205 regulate epithelial to mesenchymal transition by targeting ZEB1 and SIP1. Nat Cell Biol. 2008;10(5):593-601.

69. Park SM, Gaur AB, Lengyel E, Peter ME. The miR-200 family determines the epithelial phenotype of cancer cells by targeting the E-cadherin repressors ZEB1 and ZEB2. Genes Dev. 2008;22(7):894-907.

70. Burk U, Schubert J, Wellner U, et al. A reciprocal repression between ZEB1 and members of the miR-200 family promotes EMT and invasion in cancer cells. EMBO Rep. 2008;9(6):582-589.

71. Korpal M, Lee ES, Hu G, Kang Y. The miR-200 family inhibits epithelial-mesenchymal transition and cancer cell migration by direct targeting of E-cadherin transcriptional repressors ZEB1 and ZEB2. J Biol Chem. 2008;283(22):14910-14914.

72. Yang S, Banerjee S, de Freitas A, et al. Participation of miR-200 in pulmonary fibrosis. Am J Pathol. 2012;180(2):484-493.

73. Xiong M, Jiang L, Zhou Y, et al. The miR-200 family regulates TGF- $\beta 1$-induced renal tubular epithelial to mesenchymal transition through Smad pathway by targeting ZEB1 and ZEB2 expression. Am J Physiol Renal Physiol. 2012;302(3):F369-F379.

74. Wang B, Koh P, Winbanks C, et al. miR-200a prevents renal fibrogenesis through repression of TGF- $\beta 2$ expression. Diabetes. 2011;60(1): 280-287.
75. Oba S, Kumano S, Suzuki E, et al. miR-200b precursor can ameliorate renal tubulointerstitial fibrosis. PLoS ONE. 2010;5(10):e13614.

76. Vettori S, Gay S, Distler O. Role of microRNAs in fibrosis. Open Rheumatol J. 2012;6:130-139.

77. van Rooij E, Sutherland LB, Qi X, Richardson JA, Hill J, Olson EN. Control of stress-dependent cardiac growth and gene expression by a microRNA. Science. 2007;316(5824):575-579.

78. Wei J, Feng L, Li Z, Xu G, Fan X. MicroRNA-21 activates hepatic stellate cells via PTEN/Akt signaling. Biomed Pharmacother. 2013;67(5): 387-392.

79. Cushing L, Kuang PP, Qian J, et al. miR-29 is a major regulator of genes associated with pulmonary fibrosis. Am J Respir Cell Mol Biol. 2011;45(2):287-294.

80. Sekiya Y, Ogawa T, Yoshizato K, Ikeda K, Kawada N. Suppression of hepatic stellate cell activation by microRNA-29b. Biochem Biophys Res Commun. 2011;412(1):74-79.

81. Roderburg C, Urban GW, Bettermann K, et al. Micro-RNA profiling reveals a role for miR-29 in human and murine liver fibrosis. Hepatology. 2011;53(1):209-218.

82. Pogribny IP, Starlard-Davenport A, Tryndyak VP, et al. Difference in expression of hepatic microRNAs miR-29c, miR-34a, miR-155, and $\mathrm{miR}-200 \mathrm{~b}$ is associated with strain-specific susceptibility to dietary nonalcoholic steatohepatitis in mice. Lab Invest. 2010;90(10): 1437-1446.

83. Lan HY, Mu W, Tomita N, et al. Inhibition of renal fibrosis by gene transfer of inducible Smad7 using ultrasound-microbubble system in rat UUO model. J Am Soc Nephrol. 2003;14(6):1535-1548.

84. Li T, Li D, Sha J, Sun P, Huang Y. MicroRNA-21 directly targets MARCKS and promotes apoptosis resistance and invasion in prostate cancer cells. Biochem Biophys Res Commun. 2009;383(3):280-285.

85. Zhang YK, Wang H, Leng Y, et al. Overexpression of microRNA-29b induces apoptosis of multiple myeloma cells through down regulating Mcl-1. Biochem Biophys Res Commun. 2011;414(1):233-239.

86. Velu VK, Ramesh R, Srinivasan AR. Circulating microRNAs as biomarkers in health and disease. J Clin Diagn Res. 2012;6(10): 1791-1795.

87. Glowacki F, Savary G, Gnemmi V, et al. Increased circulating miR-21 levels are associated with kidney fibrosis. PLOS ONE. 2013;8(2): e58014.

88. Argyropoulos C, Wang K, McClarty S, et al. Urinary microRNA profiling in the nephropathy of type 1 diabetes. PLoS ONE. 2013;8(1):e54662.

89. Wang G, Kwan BC, Lai FM, Chow KM, Li PK, Szeto CC. Urinary miR-21, miR-29, and miR-93: novel biomarkers of fibrosis. Am J Nephrol. 2012;36(5):412-418

90. Khella HW, White NM, Faragalla H, et al. Exploring the role of miRNAs in renal cell carcinoma progression and metastasis through bioinformatic and experimental analyses. Tumour Biol. 2012;33(1):131-140.

91. Baskerville S, Bartel DP. Microarray profiling of microRNAs reveals frequent coexpression with neighboring miRNAs and host genes. $R N A$ 2005;11(3):241-247.

92. Khella HW, Bakhet M, Lichner Z, Romaschin AD, Jewett MA, Yousef GM. MicroRNAs in kidney disease: an emerging understanding. Am J Kidney Dis. 2013;61(5):798-808.

International Journal of Nephrology and Renovascular Disease

\section{Publish your work in this journal}

The International Journal of Nephrology and Renovascular Disease is an international, peer-reviewed open-access journal focusing on the pathophysiology of the kidney and vascular supply. Epidemiology, screening, diagnosis, and treatment interventions are covered as well as basic science, biochemical and immunological studies. The journal welcomes
Dovepress

original research, clinical studies, reviews \& evaluations, expert opinion and commentary, case reports and extended reports. The manuscript management system is completely online and includes a very quick and fair peerreview system, which is all easy to use. Visit http://www.dovepress.com/ testimonials.php to read real quotes from published authors. 